\title{
Picos de pressão intra-ocular: comparação entre curva tensional diária, minicurva e medida da pressão intra-ocular às 6 horas
}

\author{
Intraocularpressure peaks:comparison between the circadian curve, diurnal curve \\ and the 6 a.m. measurement
}

\author{
Luciana Duarte Rodrigues ${ }^{1}$ \\ Maria Rosa Bet de Moraes Silva ${ }^{2}$ \\ Silvana Artioli Schellini ${ }^{3}$ \\ Edson Nacib Jorg'e ${ }^{4}$
}

Residente do Departamento de Oftalmologia, Otorrinolaringologia e Cirurgia de Cabeça e Pescoço da Faculdade de Medicina de Botucatu da Universidade Estadual Paulista - UNESP.

${ }^{2}$ Professora Assistente Doutora do Departamento de Oftalmologia, Otorrinolaringologia e Cirurgia de $\mathrm{Ca}$ beça e Pescoço da Faculdade de Medicina de Botucatu da Universidade Estadual Paulista - UNESP.

${ }^{3}$ Professora Livre-Docente do Departamento de Oftalmologia, Otorrinolaringologia e Cirurgia de Cabeça e Pescoço da Faculdade de Medicina de Botucatu da Universidade Estadual Paulista - UNESP.

${ }^{4}$ Professor Assistente Doutor do Departamento de Oftalmologia, Otorrinolaringologia e Cirurgia de Cabeça e Pescoço da Faculdade de Medicina de Botucatu da Universidade Estadual Paulista - UNESP.

Endereço para correspondência: Maria Rosa Bet de Moraes Silva, DEP. OFT/ORL/CCP - Faculdade de Medicina de Botucatu - UNESP - Botucatu (SP) CEP 18618-000

E-mail: rosabet@fmb.unesp.br

Recebida para publicação em 14.10.2002

Versão revisada recebida em 18.03.2003

Aprovação em 30.05.2003

Nota Editorial: Pela análise deste trabalho e por sua anuência na divulgação desta nota, agradecemos ao Dr. Riuitiro Yamane.

\begin{tabular}{l} 
RESUMO \\
\hline Introdução: A pressão intra-ocular (Po) é o fator de risco isolado mais \\
importante para o desenvolvimento do glaucoma primário de ângulo \\
aberto(GPAA). O controle da Poé o objetivo principal da terapia antiglau- \\
comatosa até o momento. A curva tensional diária (CTD) é de grande \\
importância para o diagnóstico e seguimento do glaucoma primário de \\
ângulo aberto. Métodos simplificados como a minicurva têm sido utiliza- \\
dos em seu lugar por serem mais práticos. Objetivos: Comparar curva \\
tensional diária, minicurva e medida isolada às 6 horas quanto à detecção \\
de picos pressóricos e verificar a influência da variação postural na medida \\
das 6 horas da manhã. Métodos: Sessenta e quatro pacientes (126 olhos) \\
com glaucoma primário de ângulo aberto ou suspeita de glaucoma foram \\
submetidos à curva tensional diária. A minicurva considerou as medidas \\
das 9, 12, 15 e 18 horas da mesma curva tensional diária. A medida das 6 \\
horas foi realizada no escuro, com opaciente deitado, utilizando o tonômetro \\
de Perkins. Logo após, foi feita nova medida, com o paciente sentado, \\
usando o tonômetro de Goldmann. A Po média e a ocorrência de picos \\
(Po 21 mmHg) da curva tensional diária e minicurva foram comparados, \\
assim como o horário de ocorrência dos picos. Resultados: APo média foi \\
maior no glaucoma primário de ângulo aberto do que nos suspeitos tanto \\
na minicurva como na curva tensional diária Quando comparadas, a curva \\
tensional diária apresentou médias de Po maiores que a minicurva. A \\
medida das 6 horas foi maior quando feita com o paciente deitado. A \\
minicurva não detectou $60,42 \%$ dos picos nos pacientes com glaucoma \\
primário de ângulo aberto e $88,24 \%$ dos picos nos suspeitos. Conclusões: \\
1. A curva tensional diária detectou mais picos pressóricos do que a \\
minicurva; 2. A média de Po das 6 horas foi maior com o paciente deitado; \\
3. A Po das 6 horas com o paciente deitado foi maior do que a Po média da \\
curva tensional diária e da minicurva.
\end{tabular}

Descritores: Pressão intra-ocular; Glaucoma de ângulo aberto/prevenção e controle; Tonometria; Ritmo circadiano/fisiologia; Hipertensão ocular; Campos visuais

\section{INTRODUÇ̃̃̃O}

Vários estudos apontam a pressão intra-ocular (Po) como o fator de risco isolado mais importante para o desenvolvimento do glaucoma primário de ângulo aberto (GPAA) ${ }^{(1-2)}$. Apesar de alguns autores questionarem o benefício da redução da Po no GPAA ${ }^{(3-4)}$, o controle da Po continua sendo 
o objetivo da terapia antiglaucomatosa ${ }^{(5)}$ e a única forma de tratamento até o momento.

A Po varia durante o dia, mas ainda não foi estabelecido um ritmo circadiano em humanos ${ }^{(6)}$. Parece que os picos são, em geral, observados pela manhã $\tilde{a}^{(1)}$ e que esses picos e a Po média podem ser usados para caracterizar o perfil pressórico em um indivíduo ${ }^{(7)}$. Picos de Po não detectados no horário da consulta podem ser os responsáveis pela progressão do GPAA.

A curva tensional diária (CTD) impõe-se, portanto, como importante método propedêutico tanto para diagnóstico quanto para seguimento.

No entanto, a dificuldade prática em realizar a CTD completa levou ao emprego de métodos que pudessem substituí-la ou simplificá-la, como a Minicurva e uso de testes como o da sobrecarga hídrica. A Minicurva considera medidas da Po durante as chamadas horas de trabalho, o que facilita a sua realização para médico e paciente, além de reduzir custos. O teste de sobrecarga hídrica, por sua vez, é realizado no espaço de uma hora, poupando tempo e reduzindo custos. A CTD, entretanto, é considerada o método mais fiel para se avaliar o comportamento da Po na semiologia do glaucoma ${ }^{(8)}$.

\section{OBJETIVOS}

O presente estudo teve como objetivos:

1. Comparar CTD e Minicurva, quanto à detecção de picos e média de Po;

2. Verificar a influência da variação postural na medida das 6 horas da manhã;

3. Comparar a média de Po das 6 horas (paciente deitado e sentado) com a média de Po da CDT e da Minicurva.

\section{MÉTODOS}

Foram estudados prospectivamente 126 olhos de 64 pacientes do Ambulatório de Glaucoma do Hospital das Clínicas da Faculdade de Medicina de Botucatu - UNESP, de janeiro a outubro de 2001. Os pacientes eram portadores de glaucoma primário de ângulo aberto (GPAA) ou suspeitos de glaucoma (Suspeito). Os portadores de glaucoma tinham diagnóstico confirmado por apresentarem defeitos de campo visual. Os Suspeitos apresentavam campo visual normal e estavam em investigação por apresentarem escavação suspeita do disco óptico e/ou picos pressóricos $>20 \mathrm{mmHg}$ em medidas isoladas.

Os pacientes foram divididos em 2 grupos: GPAA e Suspeitos e ambos foram ainda subdivididos conforme o olho.

Todos os pacientes foram submetidos à curva tensional diária (CTD), realizada por sete residentes de $2^{\circ}$ ano, com medidas às $6,9,12,15,18,21 \mathrm{e} 24$ horas. A Po era medida com o paciente sentado, usando o tonômetro de aplanação de Goldman, modelo R900. Às 6 horas da manhã, a medida era feita no leito, em ambiente escuro, com tonômetro de aplanação de Perkins e também com o paciente sentado, usando o tonômetro de Goldman. A diferença de tempo entre essas duas medidas era aproximadamente 5 minutos.

Foi também estudada a Minicurva de Po considerando as medidas das 9, 12, 15 e 18 horas, obtidas da mesma CTD do paciente.

Foram também analisados os horários de ocorrência dos picos de Po, considerando-se como picos valores de $\mathrm{Po} \geq 21 \mathrm{mmHg}$.

O grupo Suspeito era formado por 29 pacientes (57 olhos), sendo 18 do sexo feminino e 11 do sexo masculino. A média de idade foi 52,21 $\pm 13,94$ anos, variando de 24 a 77 anos. Quanto à raça, havia 22 brancos e 7 negros ou pardos. Havia 4 portadores de diabetes melitus e 8 portadores de hipertensão arterial sistêmica.

O grupo dos portadores de glaucoma (GPAA) era formado por 35 pacientes ( 68 olhos), sendo 18 do sexo feminino e 17 do sexo masculino. A média das idades foi de $65,21 \pm 10,46$ anos, variando de 41 a 87 anos. Quanto à raça, 24 eram brancos, 10 negros ou pardos e 1 amarelo. Havia 9 portadores de diabetes melitus e 16 portadores de hipertensão arterial sistêmica.

Foram excluídos, um olho do grupo Suspeito e dois olhos do grupo GPAA por apresentarem cavidade anoftálmica (dois olhos) e phitisis bulbi (um olho).

Nenhum dos Suspeitos usava medicação antiglaucomatosa; entre os portadores de glaucoma, apenas 16 não estavam medicados. Dos 54 medicados, 33 usavam uma medicação apenas (betabloqueador), 17 usavam duas medicações (betabloqueador e inibidor tópico da anidrase carbônica ou alfaagonista) e 4 usavam três medicações (a terceira era um análogo de prostaglandina). Havia 14 olhos já submetidos à trabeculectomia.

Os dados foram submetidos à análise de Perfil para a comparação das médias entre grupos e quanto aos tipos de avaliação da Po (CTD ou Minicurva), seguida do método de comparações múltiplas de Turkey. O nível de significância utilizado foi de $5 \%(\mathrm{p}<0,05)$.

\section{RESULTADOS}

Os grupos foram homogêneos quanto à idade, sexo $(\mathrm{p}=0,39)$, raça $(\mathrm{p}=0,35)$ e presença de doenças sistêmicas como diabetes melitus $(\mathrm{p}=0,41)$ e hipertensão arterial sistêmica $(\mathrm{p}=0,13)$.

Considerando-se a CTD (com a medida das 6 horas sentado), a Po média foi significativamente maior no grupo do GPAA do que no grupo Suspeito (Tabela 1). Na comparação dos olhos, a Po média do olho esquerdo foi maior para o GPAA e no olho direito para os Suspeitos $(\mathrm{p}<0,05)$. A variabilidade não diferiu estatisticamente em nenhum dos grupos (Tabela 1).

Considerando-se a CTD (com a medida das 6 horas deitado), no entanto não houve nenhuma diferença da Po média tanto entre grupos como entre olhos (Tabela 2).

Na Minicurva foi observada diferença significativa entre GPAA e Suspeitos. Comparando-se olhos apenas, os portadores de glaucoma apresentaram diferença $(\mathrm{OE}>\mathrm{OD})$. A variabilidade não apresentou diferenças entre grupos e entre olhos (Tabela 3). 


\begin{tabular}{|c|c|c|c|c|}
\hline \multirow[t]{2}{*}{ Grupo } & \multicolumn{2}{|c|}{ OD } & \multicolumn{2}{|c|}{ OE } \\
\hline & $\begin{array}{c}\text { Po } \\
M_{ \pm} S\end{array}$ & $\begin{array}{c}\mathbf{v} \\
M_{ \pm} S\end{array}$ & $\begin{array}{c}\text { Po } \\
M_{ \pm} S\end{array}$ & $\begin{array}{c}v \\
M_{ \pm} S\end{array}$ \\
\hline GPAA & $15,33 \pm 3,42^{*}$ & $1,87 \pm 0,78$ & $15,94 \pm 3,23$ & $2,15 \pm 0,99$ \\
\hline Suspeito & $14,13 \pm 2,61$ & $1,83 \pm 0,70$ & $13,67 \pm 2,74$ & $1,73 \pm 0,68$ \\
\hline
\end{tabular}

\begin{tabular}{|c|c|c|c|c|}
\hline \multirow[t]{3}{*}{ Grupo } & \multicolumn{2}{|c|}{ OD } & \multicolumn{2}{|c|}{ OE } \\
\hline & Po & $\mathbf{v}$ & Po & $\mathbf{v}$ \\
\hline & $M \pm S$ & $\mathbf{M} \pm \mathbf{s}$ & $\mathbf{M} \pm \mathbf{S}$ & $M \pm s$ \\
\hline GPAA & $15,65 \pm 3,45$ & $2,47 \pm 1,39$ & $16,53 \pm 2,72$ & $3,05 \pm 1,11$ \\
\hline Suspeito & $15,03 \pm 2,90$ & $2,92 \pm 1,27$ & $14,79 \pm 2,88$ & $2,69 \pm 1,40$ \\
\hline
\end{tabular}

\begin{tabular}{|c|c|c|c|c|}
\hline \multirow[t]{3}{*}{ Grupo } & \multicolumn{2}{|c|}{ OD } & \multicolumn{2}{|c|}{$\mathrm{OE}$} \\
\hline & Po & $\mathbf{v}$ & Po & $\mathbf{v}$ \\
\hline & $M_{ \pm} \mathbf{S}$ & $M_{ \pm S}$ & $M_{ \pm} S$ & $M_{ \pm} \mathbf{S}$ \\
\hline GPAA & $14,96 \pm 3,39$ & $1,93 \pm 1,20$ & $15,85 \pm 3,59^{*}$ & $1,81 \pm 1,14$ \\
\hline Suspeito & $13,65 \pm 2,49$ & $1,63 \pm 0,89$ & $13,32 \pm 2,75$ & $1,46 \pm 0,82$ \\
\hline
\end{tabular}

\begin{tabular}{|l|lcc|}
\hline \multicolumn{4}{|c|}{$\begin{array}{l}\text { Tabela 4. Comparação entre Minicurva e CTD (medida das } \\
\text { com paciente sentado) quanto à média de pressão intra-ocular (Po) } \\
\text { em mmHg em OD e OE }\end{array}$} \\
Grupo & Olhos & Minicurva & CTD \\
GPAA & OD & 14,96 & 15,33 \\
& OE & 15,86 & $15,94^{*}$ \\
Suspeito & OD & 13,65 & 14,13 \\
& OE & 13,32 & $13,67^{*}$ \\
\multirow{2}{*}{ * significativo para $p<0,05$} & & \\
\hline
\end{tabular}

\begin{tabular}{|lccc|}
\hline \multicolumn{4}{|c|}{ Tabela 5. Po às $\mathbf{6}$ horas deitado e sentado. Comparação dos valores } \\
de média (M) em $\mathbf{~ m m H g}$ em OD e OE \\
Grupo & Olho & Po deitado (6 h) & Po sentado $(\mathbf{6} \mathbf{~ h})$ \\
GPAA & OD & $19,58^{*}$ & 16,19 \\
& OE & $20,18^{*}$ & 16,66 \\
\multirow{2}{*}{ Suspeito } & OD & $19,59^{*}$ & 15,27 \\
& OE & $18,94^{*}$ & 15,18 \\
* significativo para $\mathrm{p}<0,05$ & & \\
\hline
\end{tabular}

Comparando-se a Po média da CTD e da Minicurva para o OD não foram observadas diferenças. Para o OE houve diferença tanto para os portadores de GPAA como para os Suspeitos (Tabela 4).

Considerando a medida das 6 horas isoladamente, os valores obtidos com o paciente deitado foram maiores do que os obtidos com o paciente sentado, para os 2 grupos e para OD e OE (Tabela 5).
Comparando-se a Po média da CTD com a Po isolada das 6 horas (paciente sentado), observou-se que a medida das 6 horas foi sempre maior tanto para os 2 grupos como para OD e OE, no entanto foi significativa apenas para OE (Tabela 6).

Comparando-se a Po média da CTD com a medida das 6 horas (paciente deitado), observaram-se também valores maiores com a medida das 6 horas que foram todos significativos (Tabela 7). Comparando-se a Po da Minicurva com a Po isolada das 6 horas (paciente sentado e deitado) houve diferença significativa entre os grupos e entre os olhos sendo sempre maior às 6 horas (Tabela 8).

Os picos de Po (considerando ambos os olhos) no grupo GPAA ocorreram em 41,67\% na medida das 6 horas (paciente deitado). Nos horários da Minicurva ocorreram 39,58\% dos picos, sendo que esta não foi capaz de detectar $60,42 \%$ dos mesmos. No grupo Suspeito, a medida das 6 horas detectou $82,35 \%$ dos picos. A Minicurva neste grupo detectou $11,75 \%$ portanto deixou de detectar $88,24 \%$ dos picos (Tabela 9; Gráficos 1 e 2).

\section{DISCUSSÃO}

O propósito para a realização da CTD é detectar picos pressóricos não observados na rotina e que poderiam explicar a progressão do dano glaucomatoso ${ }^{(9-10)}$. Cerca de $1 / 3$ dos pacientes com Po aparentemente satisfatória em medidas de rotina apresentam picos ao longo das 24 horas $^{(11)}$. 


\begin{tabular}{|lccc|}
\hline \multicolumn{4}{|l}{ Tabela 6. Comparação entre a Po média da CTD e Po às 6 horas } \\
(paciente sentado). Média dos valores em mmHg em OD e OE \\
Grupo & Olho & CTD & Po 6 horas (sentado) \\
GPAA & OD & 15,33 & 16,19 \\
& OE & 15,94 & $16,66^{*}$ \\
\multirow{2}{*}{ Suspeito } & OD & 14,13 & 15,27 \\
& OE & 13,66 & $15,18^{*}$ \\
$*$
\end{tabular}

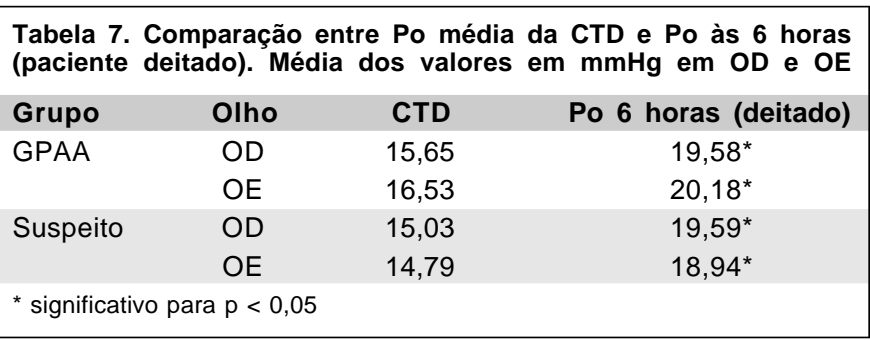

\begin{tabular}{|c|c|c|c|c|}
\hline Grupo & Olho & Minicurva & $\begin{array}{l}\text { Po } 6 \text { horas } \\
\text { (deitado) }\end{array}$ & $\begin{array}{l}\text { Po } 6 \text { horas } \\
\text { (sentado) }\end{array}$ \\
\hline \multirow[t]{2}{*}{ GPAA } & OD & 14,95 & $19,59^{*}$ & $16,19^{*}$ \\
\hline & OE & 15,85 & $20,18^{*}$ & $16,66^{*}$ \\
\hline \multirow[t]{2}{*}{ Suspeito } & OD & 13,64 & $19,59^{*}$ & $15,27^{*}$ \\
\hline & OE & 13,32 & $18,94^{*}$ & $15,18^{*}$ \\
\hline
\end{tabular}

\begin{tabular}{|c|c|c|c|c|c|c|c|}
\hline \multirow[t]{2}{*}{ Grupo } & \multicolumn{7}{|c|}{ Horário } \\
\hline & $\begin{array}{l}6 \mathrm{~h} \\
\mathrm{n} \%\end{array}$ & $\begin{array}{l}9 \mathrm{~h} \\
\mathrm{n} \%\end{array}$ & $\begin{array}{l}12 \mathrm{~h} \\
\mathrm{n} \%\end{array}$ & $\begin{array}{l}15 \mathrm{~h} \\
\mathrm{n} \%\end{array}$ & $\begin{array}{l}18 \mathrm{~h} \\
\mathrm{n} \%\end{array}$ & $\begin{array}{l}21 \mathrm{~h} \\
\mathrm{n} \%\end{array}$ & $\begin{array}{l}24 \mathrm{~h} \\
n \%\end{array}$ \\
\hline GPAA & $\begin{array}{c}20 \\
(41,67)\end{array}$ & $\begin{array}{c}5 \\
(10,42)\end{array}$ & $\begin{array}{c}5 \\
(10,42)\end{array}$ & $\begin{array}{c}5 \\
(10,42)\end{array}$ & $\begin{array}{c}4 \\
(8,33)\end{array}$ & $\begin{array}{c}3 \\
(6,25)\end{array}$ & $\begin{array}{c}6 \\
(12,5)\end{array}$ \\
\hline Suspeito & $\begin{array}{c}14 \\
(82,35)\end{array}$ & $\begin{array}{c}1 \\
(5,88)\end{array}$ & 0 & $\begin{array}{c}1 \\
(5,88)\end{array}$ & 0 & 0 & $\begin{array}{c}1 \\
(5,88)\end{array}$ \\
\hline
\end{tabular}

Neste estudo, a Po média foi maior na CTD, quando comparada com a Minicurva, nos 2 grupos. A Minicurva, quando comparada com a CTD, deixou de detectar 60,42 e 88,24\% dos picos, respectivamente entre os portadores de GPAA e os Suspeitos. Esses fatores podem ser explicados pelo horário de maior probabilidade dos picos, que ocorreram justamente às 6 horas com o paciente deitado, quando a Minicurva ainda não havia sido iniciada. A distribuição dos picos nas 24 horas concordou com trabalhos existentes que apresentaram $2 / 3$ dos picos antes do $\operatorname{almoço}^{(12)}$.

A Po média tanto da CTD como da Minicurva foi maior

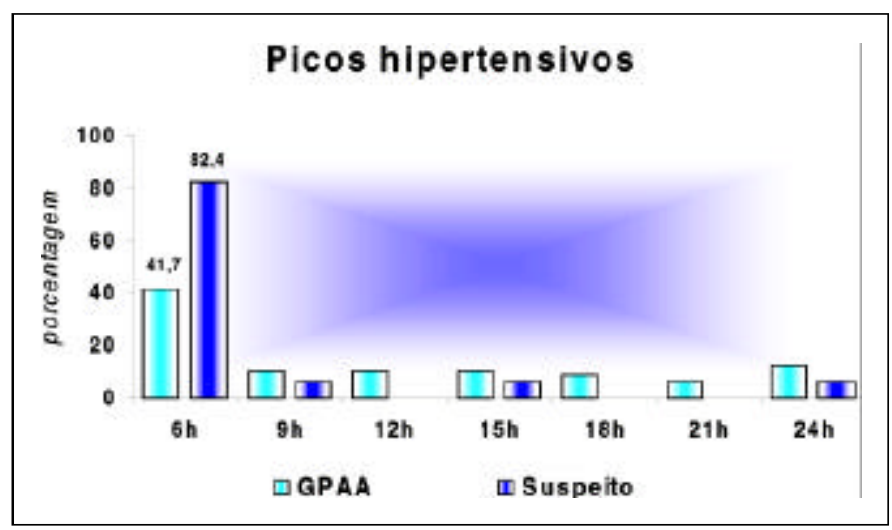

Gráfico1 - Porcentagens de picos pressóricos segundo o horário de ocorrência na CTD (paciente deitado)

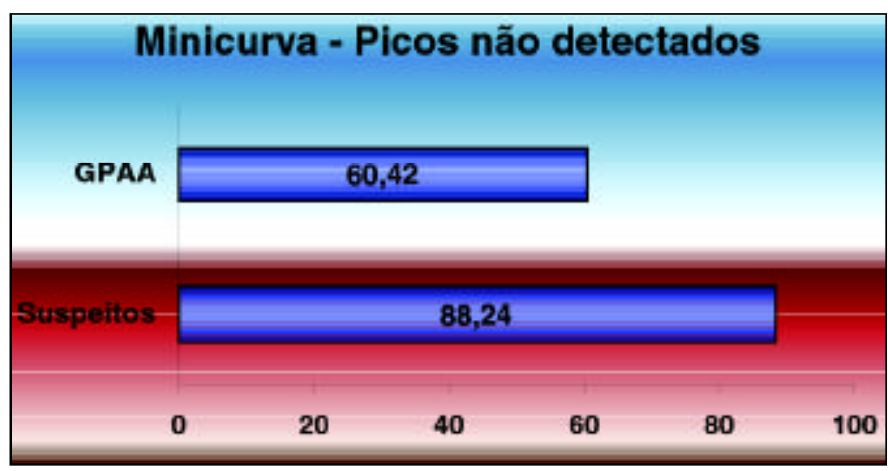

Gráfico 2 - Porcentagens de picos pressóricos não detectados na Minicurva

para o grupo de GPAA do que para o grupo Suspeito, um fato naturalmente esperado.

Vários trabalhos já demonstraram que a Po costuma ser maior pela manhã do que à noite na maioria da população ${ }^{(1,13)}$. Observamos, das diversas comparações realizadas, que a Po medida às 6 horas com o paciente deitado foi sempre maior que a Po das 6 horas com o paciente sentado, e também maior que a média dos valores obtidos da CTD e da Minicurva. Isto mostra a importância não só da medida da Po neste horário mas também da posição para detecção de picos pressóricos.

Também em relação à influência da alteração postural nos valores de Po, observando que a Po deitado é maior do que a Po sentado, nossos resultados estão de acordo com a literatura ${ }^{(11)}$. A explicação para este fato seria alterações hidrostáticas, como a elevação da pressão venosa episcleral, que ocorrem de uma posição para a outra ${ }^{(12)}$.

Os resultados observados neste estudo sugerem, portanto, que, dos métodos utilizados, o melhor para se detectar pico pressórico seria a medida das 6 horas com o paciente deitado, seguido da CTD e Minicurva.

\section{CONCLUSÕES}

1. A CTD detectou mais picos pressóricos do que a Minicurva; 
2. A média de Po da CTD foi maior que Minicurva;

3. A média de Po das 6 horas é maior com o paciente deitado;

4. A Po das 6 horas deitado foi maior do que a média de Po da CTD e Minicurva.

\section{AGRADECIMENTO}

Os autores agradecem a Professora Doutora Lídia Raquel de Carvalho do Departamento de Bioestatística do Instituto de Biociências da UNESP de Botucatu pela análise estatística.

\section{ABSTRACT}

Introduction: Intraocular pressure (IOP) is a major risk factor for the development of open angle glaucoma (OAG). Intraocular pressure control is the main target of glaucoma therapy at the moment. The 24-hour intraocular pressure curve (circadian curve-CC) is of great importance for open angle glau-coma diagnosis and follow-up. Because of practical facilities, simplified methods such as the diurnal curve (DC) have been used. Purpose: Comparison between circadian curve and diurnal curve as regards intraocular pressure peak detection and the verification of the influence of postural variation on $6 \mathrm{a} . \mathrm{m}$. measurement. Methods: Sixty-four open angle glaucoma or suspected glaucoma patients (126 eyes) were submitted to circadian curve. Diurnal curves considered 9 a.m. - noon - 3 p.m. 6 p.m. measurements of the circadian curve. At 6 a.m., measurements were performed in the dark, while patients were lying, with the Perkins tonometer. Then, another measurement was taken, with the Goldmann tonometer with the patients seated. Mean intraocular pressure and intraocular pressure peaks in the circadian and diurnal curves were compared, as well as the time when the peaks occurred. Results: Mean IOP was higher in the open angle glaucoma patients in both the circadian curve and diurnal curve. When the circadian curve and diurnal curve were compared, mean intraocular pressure was higher in the 6 a.m. circadian curve IOP measurement was higher when taken in bed. The diurnal curve did not detect $60.42 \%$ of the peaks in open angle glaucoma patients and $88.24 \%$ of the peaks in suspected glaucoma patients. Conclusions: 1 . The circadian curve detects more intraocular pressure peaks than the diurnal curve; 2. Six a.m. intraocular pressure is higher when taken in bed; 3.6 a.m. intraocular pressure in bed is higher than the mean intraocular pressure in both circadian curve and diurnal curve.

Keywords: Intraocular pressure; Glaucoma open-angle/ prevention \& control; Tonometry; Circadian rhythm/physiology; Ocular hypertension; Visual fields

\section{REFERÊNCIAS}

1. Shields MB. A pressão intraocular In: Shields MB. Glaucoma. 2 ed. São Paulo: Panamericana, 1989. p.47-73.

2. Wu YY, Leske C. Associations with intraocular pressure in the Barbados Eye Study. Arch Ophthalmol 1997;115:1572-6.

3. Konstas AGP, Mantziris DA, Cate EA, Stewart WC. Effect of timolol on the diurnal intraocular pressure in exfoliation and primary open angle glaucoma. Arch Ophthalmol 1997;115:975-9.

4. Susanna Jr R, Campagna CM. Sobrecarga hídrica - sua importância no diagnóstico do glaucoma. Rev Bras Oftalmol 1998;47:27-8.

5. Krag S, Andersen HB, Sorensen T. Circadian intraocular pressure variation with beta-blockers. Acta Ophthalmol Scand 1999;77:500-3.

6. Liu JH. Circadian rhythm of intraocular pressure. J Glaucoma 1998;7:141-7.

7. Takahashi WY, Susanna Jr R, Betinjane AJ. Reproducibilidade da curva tensional diária em dias não consecutivos. Arq Bras Oftalmol 1979;42:277.

8. Carvalho CA, Helal Jr J. Valor da prova de sobrecarga hídrica no seguimento de pacientes com glaucoma crônico simples em tratamento. Rev Bras Oftalmol 1988;47:81-4.

9. Bergea B, Bodin L, Svedbergh B. Impact of intraocular pressure regulation on visual fields in open-angle glaucoma. Ophthalmology 1999;106:997-1005; discussion p.1004-5.

10. David R, Zangwill L, Briscoe D, Dagan N, Yagev R, Yassur Y. Diurnal intraocular pressure variations: analysis of 690 diurnal curves. Br J Ophthalmol 1992;76:280-3.

11. Liu JH, Kripke DF, Hoffman RE, Twa MD, Loving RT, Rex KM, et al. Nocturnal elevation of intraocular pressure in young adults. Invest Ophthalmol Vis Sci 1998;39:2707-12.

12. Kothe AC. The effect of posture on intraocular pressure and pulsatile ocular blood flow in normal and glaucomatous eyes. Surv Ophthalmol 1994;38 Suppl:S191-7.

13. Pointer JS. Human intraocular pressure and its diurnal variation in healthy subjects. Ophthalmic Physiol Opt 1999;19(Suppl 2):S43-8.

\section{Ao enviar um artigo para publicação, leia ATENTAMENTE as instruções para autores, constante no final de cada fascículo.}

